\title{
Resistência a Antimicrobianos: a formulação da resposta no âmbito da saúde global
}

\author{
Antimicrobial Resistance: formulation of the response in the global \\ health context
}

Rafael Almeida da Silva', Beatriz Nascimento Lins de Oliveira', Luiza Pinheiro Alves da Silva², Maria Auxiliadora Oliveira', Gabriela Costa Chaves'

DOI: 10.1590/0103-1104202012602

RESUMO A Resistência a Antimicrobianos (AMR) tem se revelado como um dos maiores problemas para a saúde pública no nível global. O objetivo deste artigo foi analisar a formulação da resposta à AMR negociada no âmbito da Organização Mundial da Saúde (OMS) por seus Estados-Membros. Foram analisados os relatórios e resoluções produzidos na Assembleia Mundial da Saúde no período de 1998 a 2019. Os achados indicam que, a partir de 2014, foram estabelecidas condições de possibilidade para a aprovação do Plano de Ação Global em AMR de forma mais robusta, abrangendo o conceito de Saúde Única e envolvendo outras instâncias internacionais (FAO, OIE, OMC e PNUMA). A análise dos conteúdos e o uso de diferentes referenciais analíticos, considerando dois setores econômicos - agropecuária e indústria farmacêutica -, mostraram-se relevantes para ilustrar a complexidade da temática, reforçando sua relevância global, reconhecendo a dimensão do uso de antibióticos em animais e as lacunas em inovação tecnológica. Como a OMS, além de ser um importante agente mobilizador para a resposta à AMR no nível global, tem garantido orçamento para ações nessa área mesmo em um contexto de desfinanciamento, conclui-se que a perspectiva da saúde pública deve prevalecer na resposta à AMR.

PALAVRAS-CHAVE Saúde global. Resistência microbiana a medicamentos. Saúde Única. Indústria agropecuária. Produção de droga sem interesse comercial.

1 Fundação Oswaldo Cruz (Fiocruz), Escola Nacional de Saúde Pública Sergio Arouca (Ensp) - Rio de Janeiro (RJ), Brasil. rafaelalmmeida@gmail.com

2 Universidade Federal de Minas Gerais (UFMG)

- Belo Horizonte (MG) Brasil.

\begin{abstract}
Antimicrobial Resistance (AMR) has proved to be a major public health problem at the global level. This paper examined the formulation of the response to AMR negotiated through the World Health Organization (WHO) by its Member States. Related WHO reports and resolutions from 1998 to 2019 were analysed. The findings indicate that, from 2014 on, more robust conditions were established for approval of a Global Action Plan on AMR, encompassing the concept of One Health and involving other international entities (FAO, OIE, WTO and Unep). Content analysis and various analytical frameworks, considering two economic sectors (the livestock and pharmaceutical industries), proved relevant to illustrating the complexity of the issue, reinforcing its global importance and acknowledging the extent of antibiotic use in animals and the gaps in technological innovation. As the WHO is not only an important agent for mobilizing the response to AMR at the global level, but - despite a context of de-funding - has guaranteed a budget for action in this area, it is concluded that the public health perspective should prevail in the response to AMR.
\end{abstract}

KEYWORDS Global health. Drug resistance, microbial. One Health. Livestock industry. Orphan drug production. 


\section{Introdução}

A Resistência a Antimicrobianos (doravante, AMR, da sigla em inglês) é atualmente considerada um dos maiores problemas para a saúde pública global. Estima-se que aproximadamente quatro milhões de pessoas adquiram, anualmente, infecções associadas a cuidados de saúde na União Europeia (UE), e que cerca de 37.000 indivíduos morrem em decorrência de infecções resistentes adquiridas em ambientes hospitalares. A maioria dessas mortes (67,6\%) é provocada por bactérias multirresistentes a antibióticos'

Segundo a Organização Mundial da Saúde (OMS), a definição de AMR refere-se à capacidade de microrganismos (bactérias, fungos, vírus e parasitas) se alterarem quando expostos a antimicrobianos e de resistirem a esses medicamentos, tornando-os inefetivos ${ }^{2}$. Entretanto, muitas vezes o termo em questão é exemplificado pelo caso dos antibióticos utilizados em infecções bacterianas.

A AMR ocorre devido à interação natural entre microrganismos no meio ambiente ${ }^{3}$, mas o aumento de sua incidência pode ser decorrente de uma série de fatores, tais como o alto consumo de antimicrobianos e seu uso inadequado; a falta de informação da população; a utilização excessiva de antimicrobianos na agropecuária; e a poluição do meio ambiente causada pelo despejo de resíduos de medicamentos no solo ou na água. O problema é agravado pela ausência ou insuficiência de regulação; falta de fiscalização do consumo de antimicrobianos por parte de instituições governamentais; e pela falta de antimicrobianos inovadores decorrente do baixo investimento em Pesquisa e Desenvolvimento (P\&D) ${ }^{4-9}$.

$\mathrm{O}$ uso inadequado e excessivo de antimicrobianos na agropecuária contribui para o aumento da incidência da AMR em humanos. Esses medicamentos são utilizados na produção animal com o propósito de tratar e prevenir infecções, bem como para promover o crescimento animal, promovendo pressão seletiva nos microrganismos e tornando-os resistentes. A transmissão para humanos pode ocorrer de forma direta, mediante contato, ou indireta, no consumo do alimento e pela poluição provocada pelos resíduos biológicos agrícolas ${ }^{7}$.

Apesar da gravidade da AMR, foram raros os novos antibióticos desenvolvidos nos últimos 40 anos. Os incentivos tradicionais de mercado não foram e dificilmente serão capazes de resolver essa lacuna de inovação, especialmente num contexto de restrição de uso desses medicamentos ${ }^{10}$.

No nível internacional, em 2015, os países-membros da OMS adotaram o Plano de Ação Global sobre a AMR (Resolução WHA68.7) na Assembleia Mundial da Saúde (AMS)"1. Esse Plano foi baseado no conceito Saúde Única (One Health, em inglês), que pressupõe a relação entre saúde humana, animal e ambiental e propõe uma integração entre as diferentes áreas do conhecimento para solucionar os problemas de saúde ${ }^{12,13}$.

Dada a complexidade e a multicausalidade da AMR, as respostas para seu enfrentamento envolvem redes de regulação em várias etapas da cadeia produtiva, utilização e comercialização de antimicrobianos, com implicações para vários setores econômicos, tais como a agropecuária e a indústria farmacêutica. Considerando as múltiplas dimensões que a resposta à AMR requer, assim como os diferentes atores da sociedade e da economia diretamente envolvidos e afetados, o objetivo deste artigo é analisar a formulação da resposta à AMR negociada no âmbito da OMS por seus Estados-Membros. Parte-se do pressuposto de que, ao longo dos anos, houve uma ampliação das frentes de atuação consideradas relevantes para o enfrentamento da AMR, incluindo a necessidade de envolvimento de outros atores e instâncias multilaterais.

\section{Metodologia}

Esta pesquisa se insere no campo da análise de políticas porque reconhece o papel da formulação de propostas numa arena multilateral como a OMS. Pertencente ao sistema ONU 
(Organização das Nações Unidas), a OMS é caracterizada por ser member-driven, ou seja, dirigida pelas decisões dos seus EstadosMembros. Por meio da negociação e aprovação de resoluções na AMS, os Estados-Membros estabelecem mandatos de trabalho à própria OMS e acordam orientações para os próprios países com relação aos diferentes temas negociados ${ }^{\mathbf{1 4}}$. Apesar de as resoluções aprovadas na AMS não terem caráter vinculante, elas têm o potencial de nortear políticas, planos ou programas no nível nacional.
Os relatórios e as resoluções aprovadas são importantes fontes para análise dos principais argumentos acordados entre os países sobre o tema da AMR. Considera-se, portanto, que esses argumentos representam em si formulações de respostas à AMR que, por sua vez, podem influenciar a formulação de respostas nos níveis nacionais. Além dos relatórios e das resoluções, também foram analisados outros documentos (quadro 1), como orçamentos da OMS, abrangendo o período de 1998 a 2019 (22 anos).

Quadro 1. Lista de documentos analisados

\begin{tabular}{|c|c|}
\hline Ano & Documentos \\
\hline 1998 & $\begin{array}{l}\text { Relatório A51/915 } \\
\text { Resolução WHA51.1716 } \\
\text { Relatório Financeiro e Declarações Auditadas do período entre 1998-199917 }\end{array}$ \\
\hline 2001 & $\begin{array}{l}\text { Estratégia Global para contenção de AMR - WHO/CDS/CSR/DRS/2001.218 } \\
\text { Relatório Financeiro e Declarações Auditadas do período entre 2000-200119 }\end{array}$ \\
\hline 2005 & $\begin{array}{l}\text { Relatório A58/1420 } \\
\text { Resolução WHA58.2721 } \\
\text { Relatório Financeiro e Declarações Auditadas do período entre 2002-200322 } \\
\text { Relatório de avaliação de desempenho: Orçamento do programa 2004-200523 }\end{array}$ \\
\hline 2014 & $\begin{array}{l}\text { Relatório A67/3924 } \\
\text { Resolução WHA67.2525 } \\
\text { Plano de ação Global (draft) A67/39 Add.926 } \\
\text { Relatório de avaliação de desempenho: Orçamento do programa 2006-200727 } \\
\text { Relatório de avaliação de desempenho: Orçamento do programa 2008-200928 } \\
\text { Relatório de avaliação de desempenho: Orçamento do programa 2010-201129 } \\
\text { Relatório de avaliação de desempenho: Orçamento do programa 2012-201330 } \\
\text { Relatório de avaliação de desempenho: Orçamento do programa 2014-201531 }\end{array}$ \\
\hline 2015 & $\begin{array}{l}\text { Relatório A68/19; A68/2032 } \\
\text { Resolução WHA68.733 } \\
\text { Plano de ação Global (draft) A68/20 e A68/20 Add.111 }\end{array}$ \\
\hline 2016 & $\begin{array}{l}\text { Relatório A69/2434 } \\
\text { Plano de ação Global - Stewardship Framework - relatório do secretariado A69/24 Add.135 } \\
\text { Painel de alto nível sobre resistência a antimicrobianos (ONU)36 } \\
\text { Relatório de Resultados da OMS: Orçamento do programa 2016-201737 }\end{array}$ \\
\hline 2017 & $\begin{array}{l}\text { Relatório } A 70 / 12^{\mathbf{3 8}} \\
\text { Diretrizes sobre o uso de antimicrobianos na produção de alimentos de origem anima|39 }\end{array}$ \\
\hline 2018 & $\begin{array}{l}\text { Memorândum de entendimento entre FAO/OIE/OMS/Unep } \mathbf{4 0} \\
\text { Relatório de Resultados da OMS: Orçamento do programa 2018-201941 }\end{array}$ \\
\hline 2019 & $\begin{array}{l}\text { Follow-up to the high-level meetings of the United Nations General Assembly on health-related issues } \\
\text { Resolution and decisions of Seventy-Second World Health Assembly. Antimicrobial resistance } \\
\text { Relatório da Secretária Geral das Nações Unidas } \\
\text { 'Nu: } \\
\text { 'No time to wait: Securing the future from drug-resistant infections' }\end{array}$ \\
\hline
\end{tabular}

Fonte: Elaboração própria. Documentos disponíveis em: https://www.who.int/. 
O estudo utilizou uma triangulação de múltiplos referenciais teóricos. Adotou-se o conceito de ciclo das políticas públicas, proposto por Howlett e Ramesh (2009) (apud Mattos et al. ${ }^{45}$, envolvendo cinco fases distintas, quais sejam: montagem da agenda, formulação da política, tomada de decisão, implementação e avaliação da política. Embora esteja relacionado com as políticas públicas no nível nacional, considera-se que algumas de suas fases - formulação e implementação - possam ser úteis para analisar o papel de um processo internacional e, posteriormente, sua influência na formulação de políticas no âmbito nacional. Assim, considera-se que este estudo é um esforço de reconhecimento da dimensão internacional na formulação de políticas públicas no nível nacional.

Considerou-se a proposta de governança da saúde global, descrita por Frenk \& Moon ${ }^{46}$, para analisar a arquitetura estabelecida no processo conduzido na OMS na temática em questão. A análise da dimensão institucional desenvolvida por Sell ${ }^{47}$ também se mostrou relevante para avaliar questões da governança global (quadro 2).

Quadro 2. A dimensão institucional proposta por Sell e as funções do sistema de saúde global propostas Frenk\&Moon

Dimensão Institucional

Escopo de instituições multilaterais (arenas políticas) que estabelecem leis vinculantes e não vinculantes, declarações e resoluções a respeito da saúde global47.

Sistema de Saúde Global

Composto por grupo de atores que objetivam aprimorar a saúde global por meio do estabelecimento de regras em nível internacional. Parte do pressuposto de que nenhum país é capaz de enfrentar, sozinho, as ameaças em saúde de sua população. Sendo assim, acredita-se que é necessário desenvolver estratégias no nível internacional $\mathbf{4 6}$

Governança

A forma pela qual o sistema da saúde global é gerenciado. É composta por mecanismos formais ou informais utilizados por atores governamentais e não governamentais para coordenar estratégias de resposta aos riscos à saúde da população mundial em fóruns multilaterais $\mathbf{4 6}^{\mathbf{6}}$.

Função

Mobilização da solidariedade globa|46

Gerenciamento de externalidades entre os países $^{46}$

Produção de bens públicos globais $\mathbf{4 6}$

Stewardship (Liderança e administração)46
Descrição

Comprometimento da comunidade global com a proteção de direitos de grupos minoritários. O objetivo é reduzir as desigualdades entre as nações no que diz respeito à distribuição dos problemas de saúde $\mathbf{4 6}$

Algumas situações ocorridas em um país podem gerar efeitos em outros. Esta função propõe prevenir ou mitigar efeitos negativos promovidos à saúde no âmbito global ${ }^{\mathbf{4 6}}$

Conhecimento compartilhado pela comunidade global46

Responsável por orientar estrategicamente todo o sistema de saúde global, de maneira que as outras funções essenciais mencionadas dependem dela para funcionarem adequadamente $\mathbf{4 6}^{\mathbf{6}}$
Ferramenta

Apoio financeiro e técnico, capacitação profissional e assistência humanitária ${ }^{\mathbf{4 6}}$

Vigilância epidemiológica e compartilhamento de informações e coordenação para a preparação e resposta 46

Ferramentas de padronização internacional e orientações sobre melhores práticas. Ex: Classificação internacional de doenças e Lista de Medicamentos Essenciais ${ }^{46}$

Subfunções

A convocatória para negociação e construção de um consenso; estabelecimento de prioridades; determinação de regras para gerenciamento; avaliação dos atores e suas ações; e a defesa das causas da saúde em outras arenas políticas que possam influenciar as decisões tomadas na arena da saúde global|46

Fonte: Elaboração própria, baseado em Frenkel e Moon $\mathbf{4 6}$ e Sel|47. 
A análise dos documentos, baseada na proposta de Minayo et al. ${ }^{48}$, considerou previamente três categorias (aspectos gerais sobre AMR, inovação farmacêutica e agropecuária). A partir da leitura dos documentos, as categorias foram reorganizadas para facilitar a interpretação e a síntese dos resultados. São elas: a formulação da resposta para o enfrentamento da AMR; a nova formulação da resposta à AMR e seus desdobramentos; e a governança e as funções do sistema de saúde global aplicadas ao caso da AMR.

Como a análise se baseou em documentos que foram resultantes de negociações, reconhece-se a limitação do estudo quanto à possibilidade de identificar tensões entre os países em aspectos sensíveis da temática ao longo dos processos de negociação. Considerou-se como 'processo de formulação' a evolução, ao longo do tempo, de conteúdos incluídos na resposta à AMR, assim como o envolvimento de outras instituições multilaterais.

\section{Resultados e discussão}

\section{A formulação da resposta para o enfrentamento da AMR}

O período de 1998 a 2013 foi reconhecido como o de formulação da resposta para o enfrentamento da AMR no âmbito da OMS, quando o tema foi reconhecido como um problema de saúde pública e houve discussões sobre as ações a serem tomadas para lidar com o problema. Foi possível organizar o período em três fases distintas, descritas a seguir (figura 1).

Figura 1. Linha do tempo da formulação da resposta à AMR

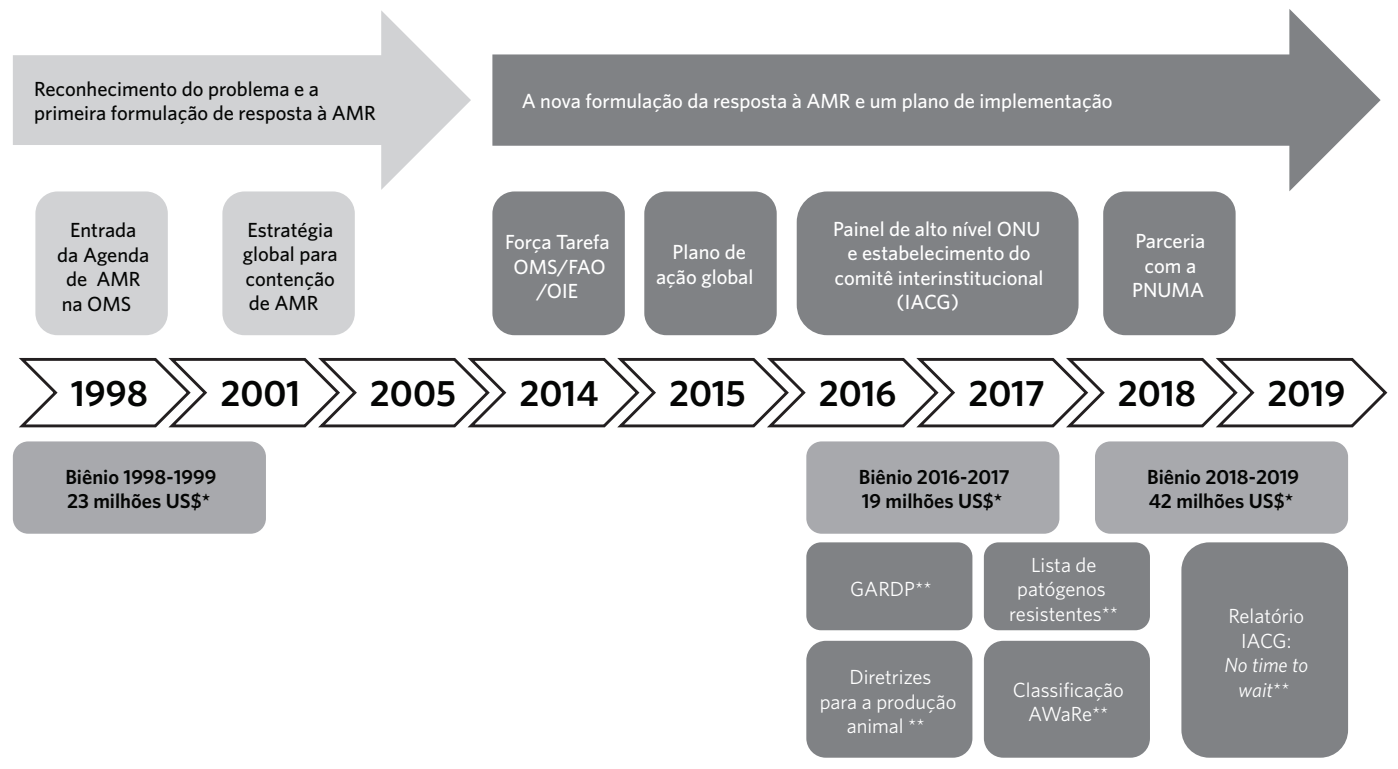

Fonte: Elaboração própria a partir da análise dos documentos.

*Orçamento Bienal.

${ }^{\star \star}$ Resposta no nível internacional.

Organização Mundial da Saúde (OMS), Organização Mundial de Saúde Animal (OIE), Organização das Nações Unidas para Alimentação e Agricultura (FAO), Organização das Nações Unidas (ONU), Programa das Nações unidas para o Meio Ambiente (PNUMA).

Parceria Global para Pesquisa e Desenvolvimento de Antibióticos (GARDP), Lista de antibióticos de acordo com o nível de resistência (Classificação AWare). 


\section{Período de 1998 a 2001: reconhecimento do problema e primeira formulação da resposta à AMR}

A AMR aparece pela primeira vez na agenda da OMS em 1998, com a discussão de um Relatório ${ }^{15}$ que resultou na aprovação da Resolução (WHA 51.17) ${ }^{16}$ na AMS. No biênio 1998-1999, foi aprovado um orçamento específico para esse tema na organização de cerca US\$23 milhões ${ }^{17}$.

Essa resolução menciona que o uso de antimicrobianos em humanos ou animais, independentemente da quantidade ou finalidade de uso, promove pressão seletiva nas bactérias, tornando-as resistentes a tais medicamentos, o que favorece a disseminação da $\mathrm{AMR}^{16}$. Reconheceu-se que a responsabilidade pelo problema era da medicina humana e da medicina veterinária, pois os medicamentos também são utilizados na produção animal e na agricultura ${ }^{15}$. Recomendou-se que governos desenvolvessem estratégias para reduzir o consumo de antibióticos em seres humanos e na produção animal15,16.

Uma das possíveis explicações para que o tema entrasse na agenda da OMS em 1998 foi o fato de que, naquele ano, a União Europeia (UE) passou por um processo de revisão de suas normas comunitárias referentes ao uso de antibióticos na produção animal49. Segundo Castanon $^{49}$, a Suécia foi o primeiro país europeu a banir o uso de promotores de crescimento a base de antibióticos, e sua entrada na UE, em 1995, trouxe o tema ao debate naquele bloco. Ainda em 1995, Alemanha e Dinamarca baniram algumas classes terapêuticas de antibióticos de uso animal, ajudando a projetar o tema no âmbito do bloco. No final da década de 1990, normativas europeias foram alteradas para aumentar a restrição do uso de antibióticos como promotores de crescimento em animais ${ }^{49}$.

Com relação aos aspectos relacionados à inovação, a Resolução afirma que o tratamento de infecções resistentes a antimicrobianos é dificultado pela falta de agentes efetivos para alguns casos e pelos preços proibitivos dos antimicrobianos de nova geração ${ }^{15}$. Muitos países não eram capazes de arcar com o preço de medicamentos inovadores, e os antimicrobianos existentes eram prescritos de forma irracional. Entre as soluções propostas estava o fortalecimento das leis de patentes. Nessa resolução, não estão previstas recomendações aos países relacionadas à lacuna de inovação. Apenas recomenda-se que a OMS colabore com o compartilhamento de conhecimento entre o setor público, a academia e a indústria, e encoraje a promoção da P\&D na área ${ }^{17}$.

Em 2001, a OMS publicou a Estratégia Global para contenção de AMR (WHO/CDS/ CSR/DRS/2001.2) ${ }^{\mathbf{1 8}}$, apresentando um conjunto de intervenções para retardar o surgimento e reduzir a disseminação de microrganismos resistentes a antimicrobianos.

Quanto à produção animal, o documento abordou a relação entre o sistema de produção de alimentos e a disseminação de AMR. O aumento da população mundial gerou o crescimento da demanda por alimentos de origem animal, e, para suprir tal necessidade, houve uma transformação do modelo de produção animal para o modelo intensivo, criando um ambiente propício à disseminação de doenças infecciosas entre os animais, sendo, então, os antibióticos utilizados no tratamento, na prevenção de doenças e também na promoção do crescimento animal18.

A OMS reconheceu a existência da relação entre o uso de antibióticos em animais e AMR em humanos com base em evidências que relataram o aparecimento de cepas bacterianas resistentes a antibióticos em humanos após a introdução desses medicamentos na produção de alimentos de origem animal. Países como França, Alemanha, Irlanda, Países Baixos e Rússia relataram a diminuição de suscetibilidade de algumas cepas a antibióticos após a liberação de seu uso na produção animal ${ }^{18}$.

Reconhecendo que a AMR é um problema complexo e multifatorial, a proposta prevista no documento de $2001^{18}$ envolve uma estratégia intersetorial com a colaboração de 
diversos setores da sociedade, como médicos; médicos veterinários; farmacêuticos e outros profissionais da saúde; produtores agropecuários; indústria farmacêutica; sociedade civil; governos e outros atores interessados ${ }^{\mathbf{1 8}}$. Nesse documento, também foi proposta a adoção de legislações para controlar o consumo de antibióticos na produção animal, tais como a obrigatoriedade da prescrição veterinária para dispensação de antibióticos ${ }^{18}$.

Sobre os aspectos relacionados à inovação tecnológica, o documento de 2001 reafirma que o risco de inexistência de terapias efetivas nos próximos anos é causado por uma lacuna de inovação para antimicrobianos. Essa lacuna é justificada pela demorada e cara atividade de P\&D e pelo fato de que as restrições no uso de novos medicamentos, indicados como última opção terapêutica, podem ter um impacto negativo nas vendas. $\mathrm{O}$ documento reconhece que as empresas devem recuperar seus gastos de P\&D e lucrar com o produto. Deve-se, então, desenvolver novos antimicrobianos e vacinas para evitar o impacto futuro da resistência, mas são necessários incentivos à $\mathrm{P} \& D$ privada nessa área ${ }^{18}$.

A Estratégia Global de $2001^{18}$ alerta para a existência de vieses em estudos de custo-efetividade, favorecendo novos agentes antimicrobianos, já que os antigos, não protegidos por patentes, não são atrativos, e existe uma carência de estudos sobre o custo ou o impacto clínico da AMR. Somado a isso, o acesso aos novos medicamentos é desigual, pois é limitado nos países menos desenvolvidos, sendo necessárias soluções para ambientes com escassez de recursos. O aumento da interação entre indústria, governo e academia é citado como uma possível solução nesse contexto ${ }^{\mathbf{1 8}}$.

Propõe-se o estabelecimento de redes internacionais de pesquisa e de cooperação para proceder à harmonização de requisitos regulatórios. É citada especificamente a interação com a indústria, por meio de incentivos inovadores, para que ela invista na P\&D de antimicrobianos. Algumas sugestões mencionadas são um fast track regulatório ou a aplicação de uma política semelhante à de doenças órfãs; a cessão de exclusividade por tempo limitado; e a proteção patentária adequada. As intervenções de combate à AMR devem ser adotadas no nível global, atreladas ao conceito de "bens públicos globais em saúde"18, e devem ser coordenadas de modo a evitar duplicação de esforços. Sugere-se, também, o estabelecimento de uma base de dados internacional de financiadores de pesquisa na área, criando, assim, um ponto de entrada único de projetos, o desenvolvimento de novos programas e o fortalecimento dos existentes ${ }^{18}$.

\section{PERÍODO DE 2002 A 2005: POUCA DISCUSSÃO SOBRE A TEMÁTICA}

Entre os anos de 2002 e 2004, o tema da AMR não foi abordado nas AMS, provavelmente em função da aprovação da Estratégia Global, em 2001, e pela expectativa de que os EstadosMembros estivessem formulando seus próprios planos de ação. Em 2005, a AMR volta a ser mencionada na AMS, mas aspectos relacionados ao uso de antibióticos na agropecuária não foram discutidos 20,21.

Com relação à inovação, o Relatório $(\mathrm{A} 58 / 14)^{20}$ ressalta novamente o alto preço dos antimicrobianos quando lançados no mercado, e são apresentadas propostas de soluções ao problema, como a redução de preços pela indústria e o uso de alternativas genéricas. Todavia, a Resolução WHA58.2721, do mesmo ano, não apresenta ações relacionadas a esse aspecto.

A menção da concorrência com medicamentos genéricos para lidar com os preços altos indica uma modificação frente às soluções propostas nos documentos anteriores, que apontam para o reforço do sistema de propriedade intelectual. É provável que essa mudança de orientação reflita o processo, também em curso na OMS, no tema de saúde pública, inovação e propriedade intelectual desencadeado principalmente com a aprovação, em 2003, da Resolução (WHA56.27) ${ }^{50}$ que estabeleceu a Comissão sobre Direitos de Propriedade Intelectual, Inovação e Saúde Pública (CIPIH) ${ }^{50}$. 
PERÍODO DE 2006 A 2013: POUCA VISIBILIDADE DA AMR NA AGENDA DA AMS

Durante o período de 2006 até 2013, pouco foi encontrado em termos de documentos acerca do tema AMR na OMS, ao passo que o tema da saúde pública, inovação e propriedade intelectual ganhou relevância entre 2003 a 2012, a partir dos desdobramentos do relatório da CIPIH ${ }^{51}$ (2006) e com a aprovação, em 2008, da Estratégia Global e Plano de Ação em Saúde Pública, Inovação e Propriedade Intelectual ${ }^{52}$ e do relatório do Grupo de Trabalho Consultivo de Especialistas em P\&D: Financiamento e Coordenação (CEWG), em 201253,54. Este buscava propostas de fontes novas e mecanismos inovadores de financiamento para estímulo à $\mathrm{P} \& \mathrm{D}$ para as necessidades em saúde que afetavam desproporcionalmente os países em desenvolvimento.

Durante os oito anos em que o tema da AMR não foi abordado de forma explícita nas AMS, foram observados avanços na implementação de uma estratégia global para AMR, tais como: o desenvolvimento de sistemas de vigilância epidemiológica integrada no continente Africano e em países da América Latina e a implementação de estratégias de enfrentamento do problema em regiões como o Mediterrâneo Oriental, o Sudeste Asiático, a UE e o Pacífico Ocidental50.

Apesar da existência do Plano de Ação em AMR, aprovado em 2001, a OMS considerou que não houve uma aceitação ampla pelos países ${ }^{50}$. Em 2014, dos 92 EstadosMembros presentes, somente 29 haviam desenvolvido estratégias nacionais de enfrentamento, entre os quais, $20 \%$ eram países em desenvolvimento. Sendo assim, em 2013, a Diretora-Geral da OMS estabeleceu um comitê consultivo estratégico e técnico sobre a AMR, que no mesmo ano realizou sua primeira reunião e concluiu que era necessário renovar e expandir a estratégia global para conter a $\mathrm{AMR}^{\mathbf{5 0 , 5 1}}$.

\section{Reformulação da resposta à AMR e seus desdobramentos}

\section{PERÍODO DE 2014 A 2019: FORMULAC̣ÃO DA RESPOSTA À AMR E UM PLANO DE IMPLEMENTAÇÃO}

Em 2014, o tema da AMR volta à agenda da OMS, com maior robustez e com a perspectiva de engajamento de outros atores globais, tendo sido aprovados o Relatório (A67/39) ${ }^{24}$ e a Resolução (WHA67.25) 25 .

Podem-se destacar duas mudanças propostas em 2014 com relação ao plano aprovado de 2001. A primeira foi a adoção do conceito Saúde Única como norteador da formulação do plano de ação global em AMR. Para a OMS, o conceito permite uma integração coerente, abrangente e integrada entre os níveis global, regional e nacional, envolvendo diferentes atores e setores: humano, veterinário, agrícola, ambiental e financeiro.

A segunda mudança foi o estabelecimento da força-tarefa global sobre AMR, baseada em uma colaboração tripartite entre a Organização das Nações Unidas para Alimentação e Agricultura (FAO), a Organização Mundial de Saúde Animal (OIE) e a OMS24,25.

Nesse contexto, a Diretora-Geral da OMS solicitou a elaboração do esboço de um Plano Global com o objetivo de auxiliar as decisões da Assembleia e assegurar que todos os países, especialmente aqueles em desenvolvimento, fossem capazes de responder à $\mathrm{AMR}^{\mathbf{2 4}, 25}$. Esse documento foi, então, elaborado de forma colaborativa entre as três organizações mencionadas.

Aprovado em 2014, o esboço ${ }^{26}$ apresentava as áreas prioritárias para ações em AMR, com metas e objetivos quantificáveis e planos para implementação, com definições de papéis e responsabilidades dos atores envolvidos. Também previa indicadores que permitissem que o progresso pudesse ser monitorado, mensurado e replicado.

Nos aspectos da inovação, o Relatório $($ A67/39) 24 de 2014 ampliou o escopo do 
problema, ao incluir a ausência de inovação em diagnósticos. O relatório menciona o investimento insuficiente para $\mathrm{P} \& \mathrm{D}$ em tecnologias para AMR, sejam elas preventivas (vacinas), para tratamento (antimicrobianos) ou para diagnóstico. Ressalta a necessidade de incentivos para inovação na área, associados a novos modelos que apoiem a P\&D no longo prazo e que sejam sustentáveis, e recomenda que a OMS trabalhe em parceria com a indústria. A Resolução daquele ano (WHA67.25) ${ }^{25}$ cita a carência de desenvolvimento de novos agentes antimicrobianos, mencionando o relatório do CEWG e o Observatório de P\&D. Recomenda aos países encorajarem novos modelos colaborativos e de financiamento à inovação. A necessidade de fomento à $\mathrm{P} \& \mathrm{D}$ e Inovação (PD\&I) na área é citada tanto na recomendação aos países quanto no mandato da $\mathrm{OMS}^{25}$.

A lacuna de inovação tecnológica é um ponto central nas discussões sobre AMR. A partir de 2003, a temática inovação e propriedade intelectual ganha relevância na OMS. Observa-se a inserção de argumentos e ações relacionados a esta questão de saúde pública nos documentos lançados a partir de 2014.

Em 2015, na $68^{\text {a }}$ AMS, reafirmou-se a importância da Tripartite (OMS/FAO/OIE) e mencionou-se o interesse em comum das instituições no enfrentamento da $\mathrm{AMR}^{\mathbf{3 2}}$. Naquele ano, a Tripartite desenvolveu um novo Plano de Ação Global em AMR ${ }^{11}$, e foi convocada uma reunião com o Secretário-Geral da $\mathrm{ONU}$ para realizar uma reunião de alto nível sobre AMR em $2016^{33}$.

No mesmo ano, a Resolução aprovada (WHA68.7) solicitou que todos os EstadosMembros adaptassem o Plano de Ação Global em AMR às suas prioridades nacionais. Também determinou que até a $70^{\mathrm{a}} \mathrm{AMS}$, em 2017, todos os planos dos países estivessem alinhados ao plano da $\mathrm{OMS}^{11}$ e aos padrões e diretrizes estabelecidos por outras instâncias internacionais, como a Comissão do Codex Alimentarius, FAO e OIE ${ }^{33}$.

Em 2016, a OMS publicou o Relatório $(\mathrm{A} 70 / 12)^{35}$, além de um Arcabouço Global para
Desenvolvimento e Administração do combate à Resistência a Antimicrobianos (Global Framework for Development \& Stewardship to Combat Antimicrobial Resistance) - produzido em conjunto por OMS, FAO e OIE. O Arcabouço indica a necessidade de apoiar o desenvolvimento, o controle, a distribuição e a utilização adequada de novos medicamentos antimicrobianos, ferramentas de diagnóstico, vacinas e outras intervenções, buscando ampliar a promoção do acesso a medicamentos antimicrobianos existentes e novos e tendo em conta as necessidades de todos os países e em consonância com o Plano de Ação Global ${ }^{35}$.

Em 2016, o tema da AMR ultrapassa a arena da saúde com a aprovação da Declaração Política da Reunião de Alto Nível da Assembleia Geral da ONU sobre AMR ${ }^{36}$. $O$ documento reafirma que o plano de ação global em AMR deve ser elaborado pela OMS em colaboração com a FAO, a OIE e a ONU, e representa o compromisso de Chefes de Estado e de Governos para desenvolver um Plano de Ação multisetorial considerando o conceito Saúde Única ${ }^{36}$.

Nesse documento, foi mencionado o desafio que a AMR representa para se alcançarem os Objetivos de Desenvolvimento Sustentável estabelecidos na Agenda 2030. Estabelece, também, o Grupo de Coordenação Interinstitucional sobre resistência a antimicrobianos (IACG, na sigla em inglês). O Grupo foi copresidido pela OMS e pela Secretaria Geral da ONU, e participavam outras instituições-parte do sistema ONU, diversas organizações internacionais e representantes da sociedade civil, do setor privado e especialistas de diversas áreas relacionadas à AMR. Seu principal objetivo foi produzir um relatório que auxiliasse a tomada de decisão sobre AMR na $73^{\circ} \mathrm{AMS}^{36}$. Em 2019, o Grupo publicou o relatório 'No time to wait: securing the future form drug-resistant infections' $\mathbf{4 4}^{\mathbf{4}}$.

Diferentemente dos anos anteriores, em 2016 e 2017 não houve resolução aprovada sobre AMR na AMS, já que em 2015 foi aprovado o Plano de Ação Global em AMR. Em 2018, 
a Tripartite firmou acordo de colaboração com o Programa das Nações Unidas para o Meio Ambiente (PNUMA, na sigla em português) (figura 1) para o enfrentamento da AMR com uma interface multissetorial (humana-animal-meio ambiente) ${ }^{40}$.

Após 2015, a cada AMS houve atualização dos países quanto à adoção do Plano de Ação Global pelos Estados-Membros. Em 2017, 67 países haviam desenvolvido seus planos de enfrentamento ${ }^{38}$, enquanto, em 2019, foram 117 países. No entanto, somente a metade deles estabeleceu um comitê multissetorial com representantes de diversas áreas, como é o exemplo do Brasil13. Não houve alteração no número de países que ainda estavam desenvolvendo seus planos, que permaneceu em 62 no período de $2017^{38}$ a $2019^{42}$.

Na AMS de 2019, os países reafirmaram o compromisso de continuar aumentando os esforços para adotar o Plano de Ação Global em AMR no nível nacional43. A resolução abordou a questão do uso racional de antibióticos em humanos e animais, mencionando as diretrizes clínicas para o uso de antibióticos importantes para a saúde humana ${ }^{\mathbf{4 3}}$ (Classificação 'AWaRE'). Com relação à P\&D\&I, ficou acordado que os países iriam apoiar a transferência voluntária de tecnologia para prevenir e controlar a $\mathrm{AMR}^{43}$.

Outras iniciativas lideradas pela OMS no período incluem a constituição da Parceria Global para P\&D de Antibióticos (GARDP) em 2016 e a publicação da lista de patógenos resistentes e a aprovação de diretrizes e melhores práticas para a produção animal em 2017 (figura 1 e quadro 3).

No que diz respeito ao orçamento da OMS específico para AMR, para os biênios 20162017 e 2018-2019, foram aprovados, respectivamente, US\$19 milhões e US\$42 milhões. Chama atenção que o valor previsto para 20182019 é quase o dobro daqueles previstos em 2016-2017 e em 1998-1999 (cerca de US\$23 milhões). Esse achado indica que, apesar da crise estrutural de financiamento da $\mathrm{OMS}^{58}$, houve recursos direcionados à AMR. Também indica maior amplitude e comprometimento ao plano de 2015 frente àquele de 2001.

\section{Governança e funções do sistema de saúde global aplicadas ao caso da AMR}

Fundamentando-se no conceito de Governança Global para Saúde ${ }^{\mathbf{4}}$, constatou-se na análise dos documentos que a OMS reconhece a AMR como um problema de saúde global, pois nenhum país sozinho conseguiria conter o avanço da AMR, que vai além das fronteiras nacionais e envolve diversos setores da sociedade ${ }^{\mathbf{1 8}}$.

Fica evidente que, ao longo de toda a formulação do plano de ação global em AMR na OMS, foi pautada a questão da cooperação internacional para apoiar os países em desenvolvimento e de menor desenvolvimento relativo (LDCs) a formularem seus planos de enfrentamento da AMR. Reconheceu-se a desigualdade entre os Estados-Membros como uma questão relevante, e, por esta razão, a cooperação internacional serviu como ferramenta de suporte para aqueles menos desenvolvidos, mediante desenvolvimento de laboratórios e programas de vigilância epidemiológica e treinamento de profissionais $\mathbf{1 1 0}^{\mathbf{1 1}}$.

Tanto a Estratégia Global de $2001^{18}$ quanto o Plano de Ação Global de $2015^{\mathbf{1 8}}$ reforçam a importância da vigilância epidemiológica como um dos eixos estruturantes da resposta à AMR. Recomendou-se que cada EstadoMembro desenvolvesse um sistema nacional para monitorar o consumo de antibióticos em humanos e animais e a incidência de AMR. Ainda a respeito da vigilância epidemiológica, em 2015 foi inaugurada a plataforma do Sistema de Vigilância Global sobre a Resistência a Antimicrobianos (Glass). Essa iniciativa objetiva coletar e analisar informações dos países sobre a AMR para produzir evidências que norteiem as ações nacionais, regionais e globais para o enfrentamento da AMR. Até 2019, 105 países afirmaram ter seus próprios sistemas de 
vigilância, dos quais, 67 compartilhavam seus resultados e 48 proviam os dados brutos à iniciativa Glass ${ }^{42}$.

Entende-se o Plano de Ação Global em AMR, assim como as orientações e iniciativas relacionadas ao uso de antibióticos na agropecuária, à pesquisa, ao desenvolvimento e à inovação de novos antimicrobianos (quadro 3) como exemplos de 'bens públicos globais' ${ }^{\mathbf{4 6}}$ no contexto da AMR.

Quadro 3. Exemplos que ilustram os chamados 'bens públicos globais'55 no campo da AMR

Iniciativas desenvolvidas no âmbito global para solucionar o problema da ausência de pesquisa e desenvolvimento de novos antibióticos

\begin{tabular}{|c|c|}
\hline Iniciativa & Descrição \\
\hline $\begin{array}{l}\text { Parceria global para pesquisa e desenvolvimento de } \\
\text { antibióticos (GARDP) }\end{array}$ & $\begin{array}{l}\text { Parceria entre a OMS e a Iniciativa Medicamentos Doenças } \\
\text { Negligenciadas (DNDi, sigla em inglês), que têm como ob- } \\
\text { jetivo desenvolver novas classes terapêuticas de antibióticos } \\
\text { do medicamento } \mathbf{5 5} \text {. }\end{array}$ \\
\hline Lista de patógenos resistentes & $\begin{array}{l}\text { Lista de patógenos resistentes com prioridade para } P \& D \text { de } \\
\text { novos antibióticos. Classifica os patógenos resistentes de } \\
\text { acordo com o risco de ameaça à saúde global com objetivo } \\
\text { de guiar o processo de } P \& D \text { de novas classes terapêuticas de } \\
\text { antibióticos }{ }^{56} \text {. }\end{array}$ \\
\hline Classificação 'AWaRE' dos antibióticos & $\begin{array}{l}\text { A OMS desenvolveu um banco de dados contendo } 180 \text { clas- } \\
\text { ses de antibióticos classificado de acordo o nível de resistên- } \\
\text { cia: 'Access group'; 'Watch group'; 'Reserv Group'57. O banco de } \\
\text { dados é uma ferramenta que pode ser utilizada para guiar os } \\
\text { países no monitoramento do uso de antibióticos }{ }^{\mathbf{5 7}} \text {. }\end{array}$ \\
\hline
\end{tabular}

Iniciativas desenvolvidas no âmbito da OMS para reduzir o consumo de antibióticos na produção animal Diretrizes da OMS sobre o uso de antibióticos importantes para a saúde humana na produção animal 39

\begin{tabular}{|c|c|}
\hline Orientações & Melhores práticas \\
\hline $\begin{array}{l}\text { Redução de todas as classes de antibióticos importan- } \\
\text { tes para a saúde humana na produção animal } \mathbf{3 9}\end{array}$ & $\begin{array}{l}\text { Novos antibióticos desenvolvidos para o uso humano devem } \\
\text { ser considerados criticamente importantes para a saúde } \\
\text { humana } \mathbf{3 9} \text {. }\end{array}$ \\
\hline $\begin{array}{l}\text { Os antibióticos importantes para a saúde humana não } \\
\text { devem ser utilizados para promover o crescimento } \\
\text { animal39 }\end{array}$ & $\begin{array}{l}\text { Medicamentos que são importantes para a saúde humana } \\
\text { e que não são utilizados na produção animal não devem ser } \\
\text { utilizados para este fim no futuro }{ }^{\mathbf{9} 9} \text {. }\end{array}$ \\
\hline \multicolumn{2}{|l|}{$\begin{array}{l}\text { Os antibióticos importantes para saúde humana não } \\
\text { devem ser utilizados na produção animal para preven- } \\
\text { ção de doenças infecciosas que não forem diagnosti- } \\
\text { cadas }^{\mathbf{3 9}}\end{array}$} \\
\hline \multicolumn{2}{|l|}{$\begin{array}{l}\text { Os antibióticos classificados como criticamente im- } \\
\text { portantes (alta prioridade) para medicina humana não } \\
\text { devem ser utilizados no tratamento de animais } 39 \text {. Reco- } \\
\text { mendação condicional* }\end{array}$} \\
\hline $\begin{array}{l}\text { Os antibióticos criticamente importantes para a medi- } \\
\text { cina humana não devem ser utilizados no controle da } \\
\text { disseminação de doenças dentro da produção animal } \mathbf{3 9} \text {. } \\
\text { Recomendação condicional* }\end{array}$ & \\
\hline
\end{tabular}


A saúde global seria, portanto, o resultado do processo da formulação de políticas em diversos espaços institucionais ${ }^{46}$. Considerando o conceito de dimensão institucional proposto por Sell ${ }^{47}$, constatou-se a partir da análise dos documentos que diversas instituições multilaterais participaram do processo de formulação do plano de enfrentamento da AMR, tais como OMS, FAO, OIE, ONU, Organização Mundial do Comércio (OMC) e, posteriormente, o PNUMA (figura 2). Dessa forma, tem-se mais elementos para afirmar que o desenvolvimento do plano de resposta à AMR é resultado da articulação entre organizações com perspectivas diferentes.

Figura 2. Imagem representativa da dimensão institucional da saúde global para o caso de AMR

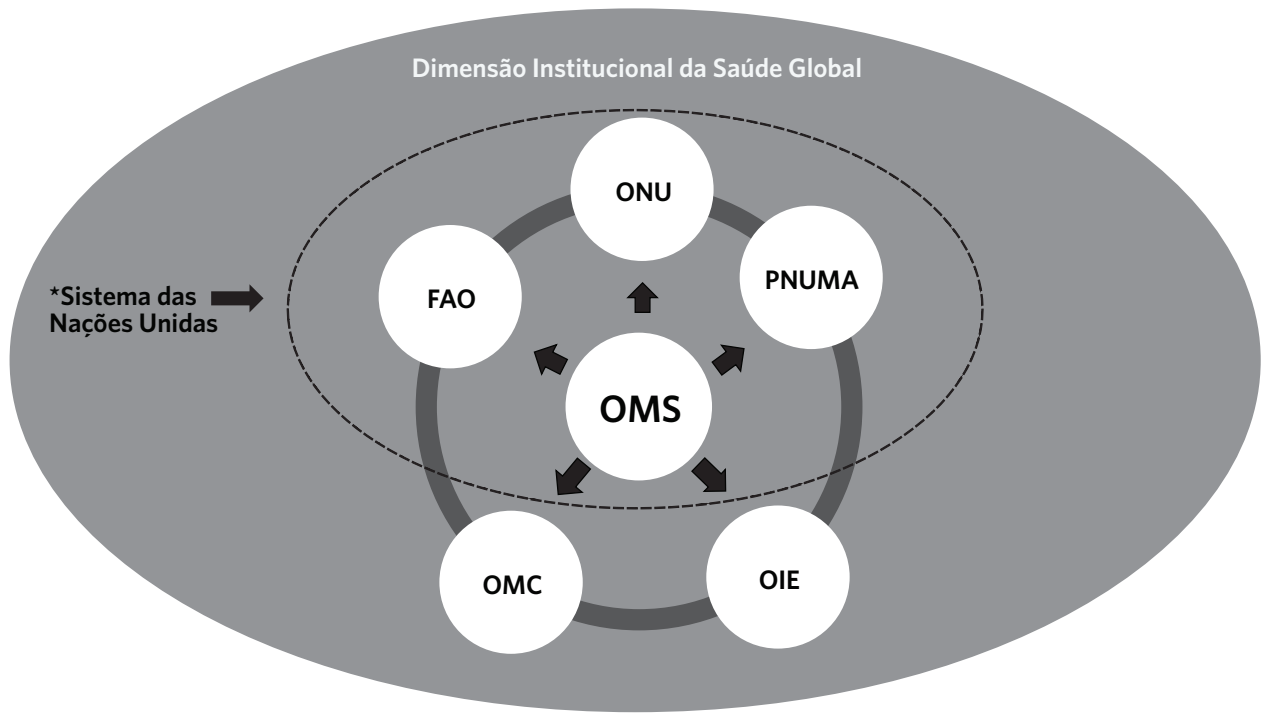

Fonte: Elaboração própria a partir da proposta teórica de Sell47.

*Sistema das Nações Unidas é composto por agências especializadas e programas institucionais.

Programa das Nações Unidas para o Meio Ambiente (PNUMA), Organização das Nações Unidas para Alimentação e Agricultura (FAO), Organização das Nações Unidas (ONU), Organização Mundial da Saúde (OMS), Organização Mundial do Comercio (OMC), Organização Mundial de Saúde Animal (OIE).

Para ilustrar essa articulação, aplicaram-se três das quatros subfunções de Stewardship (Liderança ou administração) ${ }^{\mathbf{4 6}}$, quais sejam: a defesa das causas da saúde em outras arenas políticas; a negociação e a construção de um consenso; a determinação de regras para gerenciar as dimensões intersetoriais da saúde.

Consta na resolução da $68^{\mathrm{a}}$ AMS que a parceria com a ONU foi estabelecida para juntar esforços em busca de mecanismos eficientes para implementar o plano de ação global por todas as nações, considerando as necessidades dos países em desenvolvimento ${ }^{33}$. No caso da AMR, pode-se identificar o estabelecimento da articulação entre a OMS e outros organismos multilaterais com o objetivo de defender os interesses da saúde global em outras instituições que não são responsáveis por temas relacionados à saúde, mas que podem influenciar o campo da saúde global.

No que diz respeito à negociação e à construção de um consenso sobre o uso de antibióticos na agropecuária, não foi identificada qualquer menção ao banimento do uso de antibióticos como promotores de crescimento na produção animal, provavelmente por se tratar de um tema de tensão e dissenso entre os países. No nível nacional, alguns países adotaram diferentes 
posicionamentos sobre o assunto com base em vários argumentos. Países da UE baniram os promotores de crescimento desde $2006^{59} \mathrm{e}$, recentemente (2018) ${ }^{60}$, restringiram o uso preventivo de antibióticos na produção animal60. Já alguns países em desenvolvimento, como o Brasil, permitem o uso dos promotores de crescimento $^{8,13}$.

A resolução de 2014 menciona que a parceria entre OMS, FAO e OIE foi estabelecida para manejar conflitos de interesses e, assim, viabilizar a implementação do plano de ação global em AMR 25 .

Com relação à opção entre 'banir' ou 'não banir' o uso de promotores do crescimento, o IACG chegou a um ponto intermediário, em que recomendou que alguns antibióticos classificados como criticamente importantes para a saúde humana deixassem de ser gradativamente utilizados ('phase out') ${ }^{44}$ para aquela finalidade. Tal iniciativa foi considerada no relatório como o primeiro passo em direção ao uso racional de antibióticos na produção animal ${ }^{44}$. Sendo assim, acredita-se que o estabelecimento da força-tarefa (FAO/OMS/OIE) foi fundamental para o estabelecimento do consenso no que concerne ao uso de antibióticos para promoção do crescimento.

Além da busca de consenso, os atores também estão cogitando desenvolver novas regras ou instrumentos que gerenciem as questões envolvendo AMR. Foi mencionado no relatório de 2016 o status legal que as normas, diretrizes e os códigos de prática do Codex Alimentarius e da OIE têm no âmbito da $\mathrm{OMC}^{34}$.

Quanto à subfunção da Stewardship denominada 'determinação de regras para gerenciar ações intersetoriais da saúde', destaca-se como exemplo, no relatório do IACG, o processo ainda em curso entre os países sobre a adoção de instrumentos vinculantes e não vinculantes para as medidas em AMR ${ }^{44}$.

\section{Conclusões}

Este estudo analisou a formulação da resposta à AMR no âmbito da OMS. Os achados indicam que, embora o tema esteja na agenda desde
1998, tendo sido aprovado um plano para seu enfrentamento em 2001, poucos foram os avanços percebidos do ponto de vista da adoção por parte dos Estados-Membros da OMS. Em 2014, com a adoção do conceito de Saúde Única, foram criadas as condições para a adesão de outras instituições internacionais (FAO, OIE e OMC) e aprovação do Plano de Ação Global em AMR.

Dada a complexidade e a abrangência dos fatores relacionados à AMR, era de se esperar que o desenho de uma resposta global para seu enfrentamento estaria imerso nessa complexidade, principalmente porque as necessidades em saúde pública não só tangenciam setores econômicos sensíveis - agropecuária e indústria farmacêutica - como também reforçam assimetrias entre os países. Nessa perspectiva, foi fundamental a triangulação de múltiplos referenciais analíticos que permitiram a compreensão mais extensa do objeto em estudo, reforçando sua relevância global e reconhecendo a dimensão do uso de antibióticos em animais e as lacunas em inovação tecnológica.

Como a OMS, além de importante agente mobilizador para a resposta à AMR no nível global, tem garantido orçamento para ações nessa área mesmo em um contexto de desfinanciamento, conclui-se que a perspectiva da saúde pública deve prevalecer na resposta à AMR. Resta pendente a análise do quão profunda será a adoção no nível nacional das propostas aprovadas multilateralmente para superar a amplificação da AMR e, ao mesmo tempo, assegurar que opções terapêuticas envolvendo antimicrobianos existentes e novos possam estar disponíveis àqueles que precisarem.

\section{Colaboradores}

Silva RA (0000-0002-5049-9124)*, Oliveira BNL (0000-0002-2397-5244)*, Silva LPA (0000-0002-3740-2973)*, Oliveira MA (00000003-2400-536X)*, Chaves GC (0000-0002$8347-6164) *$ contribuiram igualmente para a elaboração do manuscrito.
${ }^{\star}$ Orcid (Open Researcher and Contributor ID). 


\section{Referências}

1. European Center for Disease Prevention and Control. Antimicrobial Resistance and Health Care associated infection programme [internet]. [União Europeia]: ECDC; [acesso em 2020 set 18]. Disponível em: https://ecdc.europa.eu/en.

2. World Health Organization. Antimicrobial Resistance fact sheets- What is antimicrobial resistance? [internet]. [Genebra]: WHO. [acesso em 2017 set 10]. Disponível em: https://www.who.int/features/qa/75/en/.

3. Munita JM, Arias CA. Mechanisms of Antibiotic Resistance. In: Virulence Mechanisms of Bacterial Pathogens. Microbiol Spectr. 2016; 4(2):481-511.

4. Kaae S, Malaj A, Hoxha I. Antibiotic knowledge, attitudes and behaviours of Albanian health care professionals and patients-a qualitative interview study. J Pharm Policy Pract. 2017; 10(1):13.

5. Pavydė E, Veikutis V, Mačiulienė A, et al. Public Knowledge, Beliefs and Behavior on Antibiotic Use and Self- Medication in Lithuania. Int J Environ Res Public Health. 2015; 2(6):7002-7016.

6. Michael CA, Dominey-Howe D, Labbate M. The antimicrobial resistance crisis: causes, consequences, and management. Frontiers in public health. 2014; 2:145.

7. Roca I, Akova M, Baquero F, et al. The global threat of antimicrobial resistance: science for intervention. New microbes and new infections. 2015; 6:22-29.

8. Brasil. Decreto-lei no 8448 , de 6 de maio de 2015. Regulação da Fiscalização dos Produtos de Uso Veterinário [internet]. Diário Oficial da União. 6 Maio 2015. [acesso em 2017 nov 15]. Disponível em: http://www. planalto.gov.br/.

9. Davis MA, Hancock DD, Besser TE. Multiresistant clones of Salmonella enterica: The importance of dissemination. The Journal of laboratory and clinical medicine. 2002; 140(3):135-141.
10. United Nations. Report of the United Nations Secretary-General's High-Level Panel on Access to medicine [internet]. [Nova York]; [2016]. [acesso em 2018 set 20]. Disponível em: http://apps.who.int/medicinedocs/en/m/abstract/Js23068en/.

11. World Health Organization. Draft global action plan on antimicrobial resistance [internet]. [Genebra]: WHO; [2015]. [acesso em 2017 ago 17]. Disponível em: http://apps.who.int/gb/archive/.

12. Boqvist S, Soderqvist K, Vagsholm I. Food safety challenges and One Health within Europe. Acta Veterinaria Scandinavica. 2018, 60(1):1-13.

13. Ministério da Saúde. Resistência antimicrobiana: enfoque multilateral e resposta brasileira. In: Saúde e Política Externa: os 20 anos da Assessoria de Assuntos Internacionais de Saúde (1998-2018). [internet]. [Brasília, DF]: MS; [2018]. [acesso em 2020 maio 20]. Disponível em: www.saude.gov.br.

14. World Health Organization. Governance [internet]. [Genebra]: WHO; [data desconhecida]. [acesso em 2019 jan 20]. Disponível em: https://www.who.int/ about/governance.

15. World Health Organization. Report of regional interest adopted by the fifty-first World Health Assembly [internet]. [Genebra]: WHO; [1998]. [acesso em 2017 ago 17]. Disponível em: http://apps.who.int/gb/archive/.

16. World Health Organization. Resolution and decision of fifty-first Assembly [internet]. [Genebra]: WHO; [1998]. [acesso em 2017 ago 10]. Disponível em: http:// apps.who.int/gb/archive/.

17. World Health Organization. Financial Report and Audited Financial Statements for the period 1 January 1998- 31 December 1999. [internet]. [Genebra]: WHO; [1998]. [acesso em 2020 maio 20]. Disponível em: http://apps.who.int/gb/archive/.

18. World Health Organization. Global strategy for containment of antimicrobial resistance [internet]. [Ge- 
nebra]: WHO; [2001]. [acesso em 2017 ago 17]. Disponível em: http://apps.who.int/gb/archive/.

19. World Health Organization. Financial Report and Audited Financial Statements for the period 1 January 2000 - 31 December 2001. [internet]. [Genebra]: WHO; [2000]. [acesso em 2020 maio 20]. Disponível em: http://apps.who.int/gb/archive/.

20. World Health Organization. Report of regional interest adopted by the fifty-eight Assembly [internet]. [Genebra]: WHO; [2005]. [acesso em 2017 ago 20]. Disponível em: http://apps.who.int/gb/archive/.

21. World Health Organization. Resolution and decision of fifty-eight Assembly [internet]. [Genebra]: WHO; [2005]. [acesso em 2017 ago 17]. Disponível em: http:// apps.who.int/gb/archive/.

22. World Health Organization. Financial Report and Audited Financial Statements for the period 1 January 2002 - 31 December 2003. [internet]. [Genebra]: WHO; [2002]. [acesso em 2020 maio 20]. Disponível em: http://apps.who.int/gb/archive/.

23. World Health Organization. Performance Assessment Report: Programme Budget 2004-2005. [internet]. [Genebra]: WHO; [2004]. [acesso em 2020 maio 20]. Disponível em: http://apps.who.int/gb/archive/.

24. World Health Organization. Report of Sixty-Seventh World Health Assembly. [internet]. [Genebra]: WHO; [2014]. [acesso em 2017 ago 17]. Disponível em: http:// apps.who.int/gb/archive/.

25. World Health Organization. Resolution and decision of Sixty-Seventh World Health Assembly [internet]. [Genebra]: WHO; [2014]. [acesso em 2017 ago 20]. Disponível em: http://apps.who.int/gb/archive/.

26. World Health Organization. Draft global action plan on antimicrobial resistance. [Internet]. [Genebra]: WHO; [2014]. [acesso em 2017 ago 20]. Disponível em: http://apps.who.int/gb/archive/.

27. World Health Organization. Performance Assessment Report: Programme Budget 2006-2007. [inter- net]. [Genebra]: WHO; [2006]. [acesso em 2020 maio 20]. Disponível em: http://apps.who.int/gb/archive/.

28. World Health Organization. Performance Assessment Report: Programme Budget 2008-2009. [internet]. [Genebra]: WHO; [2008]. [acesso em 2020 maio 20]. Disponível em: http://apps.who.int/gb/archive/.

29. World Health Organization. Performance Assessment Report: Programme Budget 2010-2011. [internet]. [Genebra]: WHO; [2010]. [acesso em 2020 maio 20]. Disponível em: http://apps.who.int/gb/archive/.

30. World Health Organization. Performance Assessment Report: Programme Budget 2012-2013. [internet]. [Genebra]: WHO; [2012]. [acesso em 2020 maio 20] Disponível em: http://apps.who.int/gb/archive/.

31. World Health Organization. Performance Assessment Report: Programme Budget 2014-2015. [internet]. [Genebra]: WHO; [2004]. [acesso em 2020 maio 20] Disponível em: http://apps.who.int/gb/archive/.

32. World Health Organization. Report of sixty-eight World Health Assembly [internet]. [Genebra]: WHO; [2015]. [acesso em 2017 ago 2017]. Disponível em: http://apps.who.int/gb/archive/.

33. World Health Organization. Resolution and decision of sixty-eight Assembly [internet]. [Genebra]: WHO; [2015]. [acesso em 2017 ago 2017]. Disponível em: http://apps.who.int/gb/archive/.

34. World Health Organization. Report of sixty-nine World Health Assembly. Antimicrobial Resistance [internet]. [Genebra]: WHO; [2016]. [acesso em 2017 ago 2017]. Disponível em: http://apps.who.int/gb/archive/.

35. World Health Organization. Global action plan on antimicrobial resistance. Options for establishing a global development and stewardship framework to support the development, control, distribution and appropriate use of new antimicrobial medicines, diagnostic tools, vaccines and other interventions [internet]. [Genebra]: WHO; [2016]. [acesso em 2017 ago 20]. Disponível em: http://apps.who.int/gb/archive/. 
36. United Nations Organization. Political Declaration of the High- Level Meeting of General Assembly on Antimicrobial Resistance [internet]. [Genebra]: UN; [2016]. [acesso em 2017 set 20]. Disponível em: https://digitallibrary.un.org/record/842813.

37. World Health Organization. WHO Results Report: Programme Budget 2016-2017. [internet]. [Genebra]: WHO; [2016]. [acesso em 2020 maio 20]. Disponível em: http://apps.who.int/gb/archive/.

38. World Health Organization. Report of seventieth World Health Assembly. Antimicrobian Resistance [internet]. [Genebra]: WHO; [2017]. [acesso em 2020 maio 20]. Disponível em: http://apps.who.int/gb/archive/.

39. World Health Organization. Who guidelines on use of medically Important antimicrobials in food-producing animals [internet]. [Genebra]: WHO; [2017]. [acesso em 2017 set 15]. Disponível em: http://apps. who.int/gb/archive/.

40. World Health Organization. Memorandum of Understanding between The United Nations Food and Agriculture Organization and The World Organisation for Animal Health and The World Health Organizations. [internet]. [Genebra]: WHO; [2018]. [acesso em 2020 maio 20]. Disponível em: https://www. who.int/antimicrobial-resistance/publications/tripartite-work-plan/en/.

41. World Health Organization. WHO Results Report: Programme Budget 2018-2019. [internet]. [Genebra]: WHO; [2018]. [acesso em 2020 maio 20]. Disponível em: http://apps.who.int/gb/archive/.

42. World Health Organization. Follow-up to the high-level meetings of the United Nations General Assembly on health-related issues. [internet]. [Genebra]: WHO; [2019]. [acesso em 2020 maio 20]. Disponível em: https://apps.who.int/.

43. World Health Organization. Resolution and decisions of Seventy-Second World Health Assembly. Antimicrobial resistance. [internet]. [Genebra]: WHO; [2019]. [acesso em 2020 maio 20]. Disponível em: https:// apps.who.int/.
44. United Nations. No time to wait: securing the future from drug-resistant infections. Report to the Secretary - General of the United Nations. [internet]. [Genebra]: UN; [2019]. [acesso em 2020 maio 20]. Disponível em: http://apps.who.int/.

45. Mattos RA, Baptista, TWF. Caminhos para análise das políticas de saúde. Porto Alegre: Rede UNIDA; 2015.

46. Frenk J, Moon S. Governance challenges in global health. New England Journal of Medicine. 2013; 368(10):936-942.

47. Sell SK. The quest for global governance in intellectual property and public health: Structural, discursive, and institutional dimensions. Temp. L. Rev. 2004; 77:363.

48. Minayo CSM, Deslandes FS, Gomes R. Pesquisa social: Teoria, método e criatividade. Petrópolis: Vozes; 2009.

49. Castanon JIR. History of the use of antibiotic as growth promoters in European poultry feeds. Poultry Science. 2007; 86(11):2466-2471.

50. World Health Organization. Resolution and decision of fifty - sixth Assembly [internet]. [Genebra]: WHO; 2003. [acesso em 2019 maio 20]. Disponível em: http://apps.who.int/gb/archive/.

51. World Health Organization. Report of the Commission on Intellectual Property Rights, Innovation and Public Health [internet]. [Genebra]: WHO; [2016]. [acesso em 2019 jan 10]. Disponível em: https://www. who.int/intellectualproperty/report/en/.

52. World Health Organization. The Global Strategy and Plan of Action on Public Health, Innovation and Intellectual Property [internet]. [Genebra]: WHO; [2008]. [acesso em 2019 jan 10]. Disponível em: https://www.who.int/phi/implementation/phi_globstat_action/en/.

53. World Health Organization. Research and Development. Report of the Expert Working group [internet]. [Genebra]: WHO; [2010]. [acesso em 2019 jan 10]. Disponível em: http://apps.who.int/gb/archive/. 
54. World Health Organization. Consultative Expert Working group on Research and Development: Financing and Coordination [internet]. [Genebra]: WHO; [2012]. [acesso em 2019 maio 20]. Disponível em: http://apps.who.int/gb/archive/.

55. Drugs for Neglected Diseases Initiative. Global Antibiotic Research \& Development Partnership (GARDP) [internet]. [Genebra]. [acesso em 2019 set 10]. Disponível em: https://www.dndi.org/achievements/gar$\mathrm{dp} /$.

56. World Health Organization. Global Priority List of Antibiotic - Resistant Bacteria to Guide Research, Discovery, And Development of New Antibiotics [internet]. [Genebra]: WHO; [2017]. [acesso em 2019 set de 20]. Disponível em: https://www.who.int/.

57. World Health Organization. AWaRe Classification Antibiotics [internet]. [Genebra]: WHO; [2017]. [aces- so em 2017 set 17]. Disponível em: https://www.who. int/medicines/.

58. Ventura D, Perez F. Crise e reforma da organização mundial de saúde. Lua Nova. 2014; 92:45-77.

59. Kahn LW. One health and the politic of antimicrobial resistance. John Hopkins University Press; 2016.

60. European Parliament: veterinary medicine: another step in fighting antibiotic resistance. [internet]. [acesso em 2020 maio 10]. Disponível em: https://www.europarl.europa.eu/news/en/headlines/ society/20181018STO16580/veterinary-medicines-fighting-antibiotic-resistance.

Recebido em 11/12/2019

Aprovado em 08/06/2020

Conflito de interesses: inexistente

Suporte financeiro: Coordenação de Aperfeiçoamento de Pessoal

de Nível Superior (Capes) 